\title{
COGNITIVE OUTCOME WITH EPILEPSY AND MALFORMATIONS
}

To determine the relationship between malformations of cortical development and intellectual functioning (IQ), 54 children and adolescents with intractable epilepsy who later underwent cortical resection were studied at the Miami Children's Hospital, FL. Cortical lesions were classified as circumscribed or diffuse. Circumscribed lesions had less adverse effect on nonverbal IQ than diffuse cortical dysplasias. The same was true for verbal measures, but only with right-sided lesions. Left-sided lesions were associated with poor performance on verbal IQ tests, when compared to right-sided lesions. Younger age at onset and diffuse lesions were risk factors for greater impairment of cognitive functioning. (Klein B, Levin BE, Duchowny MS, Llabre MM. Cognitive outcome of children with epilepsy and malformations of cortical development. Neurology July (2 of 2) 2000;55:230-235). (Reprints: Bonnie Levin PhD, Department of Neurology, 1150 NW 14th St, Ste 715, Miami, FL 33136).

COMMENT. In children with intractable epilepsy, cortical diffuse dysplasias acquired in utero and early left hemisphere lesions affect cognitive functioning more adversely than circumscribed lesions or right hemisphere developmental lesions..

VALPROATE, MENSTRUAL DISORDER AND POLYCYSTIC OVARIES

Three patients treated with valproate for epilepsy, beginning at 16,17 , and 31 years of age, developed a reproductive disorder and are reported from the University of Oulu, Finland. Hyperandrogenism (elevated serum testosterone levels) and polycystic ovaries were diagnosed in all cases, and weight gain and menstrual disorder occurred in two. Lamotrigine substituted for valproate resulted in a decrease in testosterone levels in all 3, disappearance of polycystic ovaries in 2, and loss of weight and normal menstruation in 2. (Isojarvi JIT, Tapanainen JS. Valproate, hyperandrogenism, and polycystic ovaries. Arch Neurol July 2000;57:1064-1068). (Reprints: Jouko I T Isojarvi MD PhD, Department of Neurology, University of Oulu, FIN-90220 Oulu, Finland).

COMMENT. The authors advise that ovarian structure and function should be checked in women of reproductive age taking valproate for epilepsy, especially if menstrual irregularities develop during treatment. Reproductive endocrine disorders may occur with increased prevalence in women with epilepsy. See Progress in Pediatric Neurology III, 1997;p138, for a previous report by the same authors of 14 cases of valproate-induced polycystic ovarian syndrome. The incidence of this complication with valproate therapy was $64 \%$, the mean duration of treatment was 7 years, and the mean daily dose of valproate was $1070 \mathrm{mg}$.

\section{ATTENTION DEFICIT DISORDERS}

\section{GENETICS OF ADHD}

Advances in the genetics of childhood neuropsychiatric disorders over the past decade were reviewed in the literature and reported from Yale University School of Medicine, New Haven, CT. In ADHD a genetic basis is suggested by family and twin studies. A focus on dopamine neurotransmission showed that children with ADHD had a higher incidence of the high-risk variant of DRD4 than controls, but the relationship is still controversial. The dopamine transporter gene (DAT1) 